\title{
Four
}

\section{ANTECEDENT UNIVERSE COSMOLOGIES}

The Standard Model of the Big Bang assumes that the universe was created all at once out of nothing, and the question of what caused its creation is left unanswered. It does not affirm that the universe was created by nothing, only that it was created out of nothing or from a singularity, which empirically is nothing. In Steady State Cosmology, the universe as a whole is uncreated, everlasting, and spatially infinite; but individual hydrogen atoms are constantly being created out of absolutely nothing by pre-existing matter through some totally mysterious and unverified process. In Plasma Cosmology also the universe as a whole is uncreated and infinite in both time and space, but the Hubble expansion of our visible universe or metagalaxy was caused by the explosion of massive quantities of pre-existing matter and antimatter that were brought together by chance in a limited region of infinite spacetime. Assuming that the Big Bang really happened, many causal explanations of it are available in contemporary scientific cosmology.

Antecedent Universe Cosmologies affirm that the Big Bang was a rebound from the collapse (Big Crunch) of an antecedently existing universe; it was caused by an influx of energy from a preexisting world. This naturalistic account of origins was given by George Gamow's Infinite Contraction/Squeeze/ Bang/Rebound Cosmology which allows for only one contraction, one terminal squeeze, one Bang, and one rebound, and by Oscillation Cosmologies that postulate up to an infinite number of successive crunch/bang/rebounds. These two theories agree that our universe was brought into being, not by God, but by the collapse of an antecedently existing universe. In the next chapter we will explore a theory which claims that our universe was caused by indeterministic spontaneity within a pre-existing infinite Superspacetime.

Antecedent Universe theorists usually try to avoid the question of absolute origins by presupposing a naturalistic metaphysics that affirms the eternity and self-sufficiency of a system of nature that requires no God for its being, structure, or explanation. This metaphysics is not science, even if it is disguised as science. Without pretending to do natural science, throughout this chapter serious philosophical problems of intelligibility about prominent Antecedent Universe Cosmologies will be raised. If good philosophical or physical reasons are available for rejecting Antecedent Universe Metaphysics, one of many major obstacles to theistic belief has been removed.

Most Antecedent Universe Cosmologists intensely dislike creation ex nihilo and wish to avoid it because it seems to call for a Divine creation of the universe. Alan M. MacRobert, a regular contributor to Sky and Telescope, suggested in 1983 that "The idea of an oscillating universe, in which the Big Bang resulted from the recollapse of a previous phase of the universe, gained 
currency merely because it avoided the issue of creation-not because there was the slightest evidence in favor of it." As I. L. Rozental said, "The only way to account for all these facts in the framework of physical concepts, without invoking a metaphysical power, is to admit the existence of an (infinitely large?) number of universes."' It looks as if, having made a prior decision that there is no God, Oscillationists just postulate an infinite series of antecedent universes as a way to avoid metaphysical appeals to Divinity. Unfortunately, Antecedent Universe Cosmologies are just as metaphysical, non-empirical, unsimple, unsupported, and unscientific as Theism, if not more so. It really is more so, as discussions to follow will show. Atheistic cosmologists seem to assume that the world's existence without God is totally unproblematic, and that only God's existence is problematic. Not so!

\section{Gamow's Infinite Squeeze/Bang/Rebound Universe}

George Gamow thought that prior to the Big Bang an antecedent universe had been collapsing from an infinitely expanded state throughout an infinite past; our universe came into being as a rebound from the minimal-size contraction state of this antecedent universe. In 1947, Gamow wrote,

The universe is now expanding because in some previous period of its history (of which, of course, no record has been left), it contracted from infinity into a very dense state and then rebounded, as it were, propelled by the strong elastic forces inherent in compressed matter. ${ }^{3}$

Gamow never explained adequately what caused the rebound, or how to convert his concept of "elastic forces" into the formulas of physics; but he was convinced that the quantity of matter in our universe is insufficient to permit gravity to close it and bring about a second Big Squeeze. He predicted that "The distances between the neighboring galaxies are bound to increase beyond any limit, and there is no chance that the present expansion will ever stop or turn into a collapse."4 Thus, his theory of origins makes a place for only one Bang, preceded by a single but infinitely prolonged collapse, and followed by an unprecedented but infinite expansion. The expansion phase of the universe in which we happen to live will proceed forever toward an infinite fizzle. It will end with a whimper, not a Bang. About that, Gamow was probably right.

\section{Critique of Gamow's Cosmology}

George Gamow's cosmology does not survive serious critical examination for many reasons, two in particular. A. It cannot reconcile scientific knowledge with its postulate of an infinite past, and B. it cannot explain why gravity and/or mass were so different before the Big Squeeze. 


\section{A. Science and an Infinite Past}

The central problem of Infinite Contraction/Squeeze/Bang/Rebound Universe is common to all Antecedent Universe Cosmologies. It affirms, presumably on "scientific" grounds, that our universe existed in some form throughout an infinite past; but this claim cannot be confirmed scientifically. According to Gamow, "Our Universe has existed for an eternity of time."s He conjectured that the antecedent phase "contracted from infinity," but he hesitated to speculate about the preceding stage of the universe. He cautioned that "There is nothing that can be said about the pre-squeeze era of the universe," because "the maximum compression of the universe, which squeezed all matter into a uniform nuclear fluid, must have completely obliterated all the records of the earlier compressive stages." But our universe, the most impressive record of all if an antecedent crunch does indeed explain it, was not obliterated.

Gamow actually said a great deal about the Squeeze Era, despite his misleading warning that absolutely nothing can be said about it. He claimed that it existed, that it was of infinite duration, that it underwent gravitational collapse, that it was composed of matter not antimatter, that this matter was gradually compressed, that in its maximal compaction state it was 30 times larger than our sun, that this state contained "elastic forces," that it rebounded or exploded, and that our phase of the universe was created from this rebound. That is quite a lot to know to be nothing!

How did Gamow know all of these things that he was not supposed to know? If all scientific or empirical evidence was destroyed, as he conceded, then nothing that he tells us about the antecedent universe, including its existence and infinite duration, can be regarded as scientific knowledge. Good natural science must be grounded in experience as well as theory; but we experience only finite time and only one phase of the universe. We have no direct experience of infinite time, other cosmic phases, or other universes. We cannot inductively infer infinite time from finite time or many universes from one that is experientially unique. We may postulate the existence of an infinitely prolonged antecedent physical state, but no human experience could ever confirm the hypothesis directly or inductively. Belief in an infinite past is uncorroborated naturalistic metaphysics, not natural science. Naturalists complain that Theists believe in things that science cannot verify, but Naturalism suffers from exactly the same defect.

All Antecedent Universe Cosmologies are unscientific metaphysical guesses because they are totally unconfirmable. This failure is disastrous for theories offered in the name of science. Antecedent Universe Cosmologies presuppose that some universe in some form has endured perpetually through an infinite past. Yet, no empirical evidence for the infinity of space or time is available to us. Even if space and time really are infinite, we can have no scientific or empirical knowledge of it. No set of finitely enduring human scientists, 
no matter how large, can directly observe infinite space or time; and inductive logic forbids us to infer infinity from finite samples, just as it forbids us to infer white from samples of black or gold from instances of dross. As explanatory hypotheses, Antecedent Universe Cosmologies imply no observations that would confirm an Infinite World Metaphysics or that would falsify the hypothesis that space and time are immense but finite and created ex nihilo. Naturalists are welcome to their faith, but they cannot call it scientific knowledge. In fact, as we shall see, much of what we know scientifically and philosophically counts heavily against an infinite chain of successive universes.

\section{B. Gravity and Mass/Energy in the Squeeze Era}

Why does our present infinitely expanding phase of the universe lack sufficient mass/energy for gravity to shut it down, as Gamow believed, if the antecedent Squeeze Era contained enough mass/energy to contract and close it? How could the Squeeze Era include enough mass/energy to be closed by gravity if our present era does not have enough to shut it down eventually? Two possibilities suggest themselves. First, perhaps there actually was enough mass/energy in the Squeeze Era to close it, but much of it was destroyed in the Big Squeeze through some gross but unexplained violation of the law of the Conservation of Energy. Second, perhaps gravity was a much more powerful force during the Squeeze Era than it is now.

Neither option is very attractive for much the same reason. Both require radical changes in fundamental constituents of the universe during the Big Squeeze, and Gamow did not explain how gravity or mass/energy could make such fundamental changes. He did not even recognize the problem, but if he had it is doubtful that he could have solved it. In discussing Oscillation Cosmology, we will soon see that if Big Squeezes result in singularities, there can be no spatiotemporal or causal continuity from era to era. If Big Squeezes do not result in singularities, there is no good reason to think that basic laws, forces, and masses change dramatically from era to era, which creates additional problems.

In sum, by its own logic, the Infinite Contraction/Squeeze/Bang/Rebound Universe of George Gamow lies beyond the limits of scientific knowledge. Throughout an infinite past, supposedly, an antecedent universe contracted to a point of maximal compression and minimal size thirty times larger than our sun, then exploded in a Big Bang to create our open and infinitely expanding universe; but, Gamow conceded, we can know absolutely nothing about the antecedent universe. Even if one existed, all evidence for and about it would have been destroyed in the primordial fireball. Gamow clearly affirmed scientific agnosticism but inconsistently practiced unscientific gnosticism. Science cannot establish that there was an antecedent universe, much less that it collapsed throughout an infinite past. Gamow conceded that many things cannot 
be known, then pretended to know them. He did not identify the physical forces and laws that initiated the rebound; and he did not explain why the present infinitely expanding phase of the universe lacks sufficient mass/energy for gravity to close it, despite the antecedent Squeeze Era's containing enough to shut it down. No cosmologists today defend a temporally infinite universe with only one Squeeze/Bang/Rebound; but other versions of Antecedent Universe Cosmology are vigorously affirmed.

\section{Oscillation Cosmology}

According to Oscillation Cosmology, an antecedent collapsing universe ending with a Big Squeeze or a Big Crunch preceded our Big Bang. Our Big Bang was caused by a massive influx of energy from this earlier era. Did only one universe precede our own, or did an infinite number of collapsing/exploding/ expanding universes antedate us? Conceivably, only a few Bangs preceded the one that initiated our universe. If so, the question of absolute origin arises with respect to the first member of this finite series. What caused the first Bang? God, chance, or what? What selected just those few closed and oscillating universes for actualization out of an infinite number of different possible universes? Many cosmologists are convinced that some energy would be used up and lost with each oscillation, so in a finite number of rebounds, all mass/energy whatsoever would be lost. Nothing would exist today; yet, here we are!

An ancient myth explains what holds up the earth-it resis on the back of a turtle! And what holds up the turtle?-another turtle; and another; and another.... If prying minds persist, the final answer is, "The turtles go all the way down!" As does this myth, Oscillation Universe Cosmologists mistakenly believe that they can avoid the question of ultimate origins if our universe was preceded by universes that go all the way back (to infinity), each of which commenced with a Bang and ended with a Crunch. All these supposed antecedent universes start with Bangs, then expand, halt, contract, collapse, and finally explode to create new universes ad infinitum.

Oscillation Cosmologists are reluctant to calculate the duration of a complete cosmic cycle from start to finish; but a few make educated guesses. A. Karel Velan maintains that our universe is presently about 18 billion years old, that it will continue to expand for another 17.5 billion years, after which it will contract again to a point of maximal compression, bounce back, and start all over again. The whole process, he claims, takes 71 billion years from start to finish. ${ }^{8}$ Mark Israelit and Nathan Rosen calculate that "The period of oscillation of the universe is $\sim 1.2 \times 10^{12} \mathrm{yr}, ",(1.2$ trillion years). The difference in these two estimates is quite remarkable!

If our own cosmos was generated by another cosmos that antedated it, and it by another, and so on endlessly into the past, what were they like? Laws of nature, physical constants, and initial conditions could have varied immensely, 
perhaps infinitely, in antecedent universes; but one commonality is certain. All of them had to be closed universes, no matter how different they were otherwise. All of them had to collapse to create succeeding universes. The Oscillationist requirement of universal closure permanently excludes an infinite number of possible laws of nature, physical constants, and initial conditions, specifically, all that would yield an open universe. Oscillationists must deny that all possible universes (open universes, for example) are actualized by and within infinity. Once they admit this, they are in deep trouble!

Let us call a single universe falling within an extended oscillating series of successive worlds a "cosmic epoch." Alfred North Whitehead used this terminology, though he was not very specific about its scope. Under the influence of early quantum theory in the $1920 \mathrm{~s},{ }^{10}$ Whitehead thought that our own cosmic epoch is dominated by electromagnetic energy existing only in discrete quanta, and he defined a "cosmic epoch" as "the widest society of actual entities whose immediate relevance to ourselves is traceable." Our present cosmic epoch can be traced "to an aboriginal disorder, chaotic according to our ideals,"12 Whitehead believed; but there are other cosmic epochs "far beyond our immediate cosmic epoch" that are ordered very differently from our own. ${ }^{13}$ He did not know anything about Big Bang Cosmology, which was still in its infancy when these words appeared in Process and Reality in 1929; and he did not explain whether his "beyond" is to be construed spatially, temporally, or both. Mainstream Process Theology has interpreted Whitehead's wording temporally; but "widest" and "beyond" are actually spatial words, not temporal words; he did not say "oldest" or "before."

\section{A. Singularities}

Significantly, Oscillation Cosmologists disagree about whether a singularity begins and ends each cosmic epoch. This notion, very unfamiliar to most philosophers and to common sense, is very familiar to astrophysicists. A singularity is a physical state resulting from total gravitational collapse that is infinitely dense, infinitely compressed, infinitely hot, infinitely small, infinitely curved. Exactly how cosmologists interpret the reality of singularities depends upon the metaphysics they presuppose.

Would an initial singularity contain no energy at all, or would it contain a finite, or an infinite, amount of infinitely condensed energy? Exactly what cosmologists believe about the presence or absence of energy in an initial singularity varies according to whether they presuppose Oscillationism or Quantum Big Accident Cosmology (developed in Chapter Seven), which affirms that the universe arises spontaneously from absolutely nothing, not even a singularity. On the simplest interpretation of the Big Accident view, and in theistic creation ex nihilo (a world created out of nothing by God), an initial singularity is construed to be an infinitely small state of nothingness containing 
no mass, energy, space, or time at all. Many Oscillationists, by contrast, think that an initial singularity would contain all the mass/energy of an antecedent universe in an infinitely condensed state. On either view, a singularity is empirical nothingness. No one, not even God, could perceive or empirically verify claims about an entity that is infinitely small. Yet, many Oscillationists hold, a singularity is still a physically real something. Others submit that a singularity is just nothing at all, and that physical realities emerge only as a universe erupts from nothingness.

A complex view of the nature of original nothingness is defended by Quentin Smith in his debate with William L. Craig in their book titled Theism, Atheism, and Big Bang Cosmology, 1993. According to Craig, who is a creation ex nihilo Theist, the initial singularity out of which our universe erupted was just a state of physical nothingness; a singularity is an unreal and merely theoretical idealization or fiction. ${ }^{14}$ The words "infinite density" suggest the presence of something physically real; but, says Craig, "a condition of "infinite density' is precisely equivalent to 'nothing'. There can be no object in the real world that possesses infinite density, for if it had any size at all, it would not be infinitely dense."'15

By contrast, Smith, a Big Accident Atheist, contends that "Big Bang cosmology represents the singularity as a unique sort of reality, a physical reality, but it is represented as real none the less." "16 The initial singularity somehow contained matter or mass, Smith insists, "not ordinary mass, threedimensional mass, but infinitely compressed mass." " Smith does not succeed in giving empirical meaning to the notion of something that is physically real but totally devoid of physical, spatiotemporal properties.

Says Smith, the physical reality of an initial singularity means three things. First, if we extrapolate the physical quantities of our universe backwards in time, we reach a point "arbitrarily close" to the singularity in which these physical quantities "have arbitrarily high finite values". ${ }^{18}$ Second, "When the singularity is reached the values become infinite."19 Third, the initial singularity has the topology of a dimensionless point, but "It assumes, at a subsequent time, the topology of a finite three-dimensional space."20

Smith's first two points show only that the concept of "singularity" is equivalent to the notion of the ultimate limits of measurable physical quantities, but not that a singularity actually contains or consists of some mysterious, immeasurable, imperceptible, and non-extended physical mass. By definition, nothing could be a body, something physical, without being spatially or spatiotemporally extended. Smith never explains how his third state could be achieved, how an infinitely small singularity could be transformed into a finite spatiality, how a timeless state could do or become anything at a later time, or exactly what the difference is between a dimensionless point's becoming dimensioned and something's being created out of nothing. Oscillation Cosmology shares all of these problems. 
In Oscillation Cosmology, cosmic epochs are worlds that fall between Bangs in an infinite temporal series. Does each cosmic epoch begin with a dimensionless singularity of infinitely condensed mass, then expand to a maximum at which gravity overcomes the residual kinetic energy of the initial explosion, and finally recontract into another infinitely condensed singularity? Astrophysical cosmologists frequently discuss singularities that supposedly separate successive universes; but finding cosmologists who actually believe in them is very difficult. The notion that oscillating universes could be separated by singularities was first introduced and discussed by Richard C. Tolman in 1934; but it is not at all clear that Tolman himself actually believed this. ${ }^{21}$ John A. Wheeler once accepted a form of Oscillation Cosmology in which cosmic epochs are divided by intervening singularities, ${ }^{22}$ and John Gribbin ${ }^{23}$ still affirms this in combination with a wider quantum World-ensemble Metaphysics. ${ }^{24}$ In 1970, Roger Penrose and Stephen W. Hawking published a definitive proof that the theory of relativity, applied to closed universes, necessitates that they begin and end with singularities. ${ }^{25}$ Now Hawking ${ }^{26}$ seems (to some interpreters) to champion oscillating universes that avoid intermediate singularities through quantum effects that reverse cosmic contractions before universes totally collapse into nothingness. His view, which has other interpretations, will be explained later in more detail.

\section{B. Quantum Effects and Singularities}

For many reasons, a defensible Oscillation Cosmology must circumvent singularities. The existence of an antecedent universe cannot be inferred from known laws of nature if these laws break down completely at or in singularities; nor can they explain what causes an initial singularity to explode into a Big Bang. Roger Penrose says that, "A space-time singularity is, almost by definition, 'a place where the known laws of physics break down."'27

Can quantum theory rescue Oscillation Cosmology from singularities? Quantum theory embraces many oddities called "quantum effects." Two of them, quantum indefiniteness and quantum discreteness, may help Oscillationists evade initial singularities. ${ }^{28}$

Oscillationism can make a place for spatiotemporal and causal continuity between cosmic epochs if no singularities intervene, if earlier collapsing universes round off and undergo Big Bounces before they totally collapse. Quantum theory will support Oscillation Cosmologies if it can (1) eliminate initial singularities and (2) explain how all entropy or memory of preceding disorder can be lost between epochs. Two quantum effects, indefiniteness and discreteness, seem to be incompatible with initial singularities; but entropy, persisting from epoch to epoch, is a stubborn problem. 


\section{i. Quantum Indefiniteness}

According to Werner Heisenberg's Uncertainty Principle, quantum-size atomic and sub-atomic entities do not have definite simultaneous positions and velocities. It is not simply that we cannot find them together; they just never exist together. Real uncertainty, indeterminateness, and unpredictability obtain at the quantum level of physical reality, but not merely as expressions of human ignorance or of the limits of experimental investigation. Einstein defended "hidden variables" that would reconcile quantum with classical physics; but most quantum physicists agree with Heisenberg's repudiation of absolutely inaccessible physical unknowns; and many experiments in quantum mechanics now count decisively against hidden variables. Sub-microscopic quantum realities are very different in many respects from the macroscopic realities of ordinary perceptual experience.

In quantum theory, sub-atomic particles are thought to be more like waves than like billiard balls. Their existence is spread out indefinitely over a fuzzy region of spacetime, and they lack what Alfred North Whitehead called "simple location." As Stephen Hawking put it, "Particles do not have precisely defined positions and velocities but are 'smeared out' over a small region by the uncertainty principle of quantum mechanics." ${ }^{.29}$ Existing sub-atomic particles are so peculiar that we really cannot envision them. But we can try!

Applied to the universe as a whole, quantum indefiniteness implies that when a cosmic epoch contracts to the size of a quantum particle or wavicle, it cannot be squeezed down any further into a singularity because this would require it to have a definiteness that entities of that magnitude cannot and do not have. If collapse could progress indefinitely with uninterrupted continuity toward a singularity, at some point no room would be left for indefiniteness. Yet, indefiniteness is inescapable in a quantum universe. Stephen Hawking has something like quantum indefiniteness in mind when he asks, "Does time really have a beginning and, possibly, an end, as predicted by classical general relativity, or are the singularities in the big bang and the Big Crunch smeared out in some ways by quantum effects?"30

In several ways, quantum effects might prevent a contracting cosmic epoch from coalescing into a singularity.

First, if a universe expands asymmetrically due to quantum fluctuations and gravitationally retarded expansions in the loci of galaxies and superclusters, it will also contract asymmetrically; if riddled with black holes, some might explode or radiate themselves back into expansion while others are still contracting. Not all parts of a non-synchronous universe could ever meet again at a singularity. Its parts would not contract synchronously; some parts would bypass others; some would go in one direction and some in another; some would contract while others expand; and some might contract at one rate and others at other rates. It is like, "You are never going to get it all back in there again!" 
Technically, this is called "differential collapse." 31 Such an uneven collapse would make impossible the collapse of an entire universe to either a singularity or to Planck dimensions.

Second, even if all parts of a given universe do contract concurrently, they could never coalesce into a singularity because of quantum indefiniteness. If singularities are impossible or unintelligible, Oscillation Cosmologists must endorse Big Bounce universes that avoid singularities through quantum effects. A Big Bounce is a rebound from a state of minimal size and maximal compression that is larger than a singularity. Through Big Bounce strategies, Oscillation Cosmologists can avoid singularities and all their concomitant difficulties, but Oscillationism may still have other serious problems.

\section{ii. Quantum Discreteness}

Quantum theory affirms that mass/energy can exist and can be transferred only in discrete, discontinuous, minimal units or quanta. Quantum theory began in 1900 when Max Planck realized that blackbody objects, those saturated with radiant energy, release so little high frequency radiation only because energy is emitted in discrete atomistic packets or quanta, not in infinitely divisible continuous gradations. Actually existing mass/energy is not infinitely divisible into smaller and smaller real units, although it may be so divisible in our imaginations. In the twentieth century, quantum physicists learned many amazing but now well-established things about the domain of very small physical particles. Electrons can assume some orbits around atomic nuclei but not others because some potential orbits or orbital shells are forbidden by nature. The spin of quantum particles takes only certain discrete values but not others. The physical world is not an infinitely divisible continuum, except in our imaginations. Physical units are required to have definite, finite, minimal magnitudes in order to exist at all, and in order to be transferred and absorbed. Mother Nature does not permit intermediate degrees between discrete quantities. ${ }^{32}$

Quantum physics clearly excludes the actualization of all possibilities. Mother Nature tolerates only discrete quantities in multiples of Planck dimensions. Planck numbers are invariant physical constants. Many numbers that are logically possible are forbidden in physics and chemistry; others are privileged, not just in quantum theory, but presumably also within the underlying reality that the science of physics reflects.

Cosmologists who combine Oscillationism with quantum theory think that Planck spacetime $\left(10^{-43}\right.$ of a second in age and $10^{-33}$ centimeters in diameter $)$ is the ultimate minimal unit for anything physical to be at all. (See endnote 74.) If they are right, no mass/energy, and no space or time can exist that is smaller or earlier than Planck dimensions. Quantum physicists are not perfectly consistent in eschewing dimensionless entities, for quarks, electrons, and other sub-atomic particles are sometimes said to be the size of Euclidean points, which is no size 
at all ${ }^{33}$ but this embarrassment is avoided if small physical particles are regarded as tiny blotches of mass/energy or as vibrating strings with definite Planck length and magnitude. ${ }^{34}$

Quantum theory discloses that spacetime and mass/energy exist only in atomized quanta and are not physically or metaphysically divisible into infinitesimally small real units, though they are thus divisible by mathematical imagination. If they were infinitely divisible in reality, something real, something intermediate, could exist between Planck dimensions and the zero dimensions of a singularity; and a collapsing quantum universe could gradually and continuously shrink to nothing. In a quantum universe, a physical continuum is forbidden; and gravitational collapse to a singularity existing below Planck dimensions is physically impossible.

Additionally, quantum gravity may prevent a contracting universe from collapsing to a singularity. According to some theories, gravity becomes a negative or repulsive force under certain pressure conditions; and if this happens in the final stages of gravitational collapse, a universe could shrink just so far but no further before negative gravity sets in and terminates its contraction. Negative gravity may (or may not) be operable at Planck dimensions, for the theory has not been tested and confirmed. Better established is that the degeneracy pressure of compacted electrons and neutrons will prevent a universe from shrinking to a singularity, but only if, unlike our own, that universe has less than one and a half times the mass of our sun..$^{35}$ Another possibility is that if the Big Crunch is a vortex spinning all the matter in the universe at unimaginably great speeds, the powerful centrifugal force of the spin itself may forestall an ultimate collapse into a singularity. ${ }^{36}$

Quantum theory applied to the origin of the universe says that nothing can exist earlier than Planck time or with a volume smaller than Planck space. Thus, the universe was $10^{-43}$ of a second old and $10^{-33}$ centimeters in diameter when it was anything physical at all. No laws of physics could apply antecedently since nothing could exist to which physical laws apply. Planck dimensions are called "Planck's Wall," the beginning and end of all scientific knowledge, in Quantum Cosmology.

An initial singularity is inferred in non-quantum Oscillationism by extrapolating from the observed universe back to $\mathrm{T}=0$ in accord with non-quantum laws of physics. From quantum laws we cannot extrapolate back to an initial singularity because these and all other physical laws become inapplicable below Planck dimensions. Some Quantum Cosmologists disagree and suggest that the laws of quantum physics do not breakdown at or below Planck dimensions, but if they do we obviously cannot use these laws to extrapolate back either to an initial singularity, or to an antecedent universe.

Stephen Hawking does not specify exactly which quantum effects are relevant, but he may have had quantum indefiniteness and discreteness in mind in 1988 when he announced that he had changed his mind about singularities 
and was trying to persuade other physicists that "There was in fact no singularity at the beginning of the universe," that "It can disappear once quantum effects are taken into account." ${ }^{37}$ Hawking seems to think that, like the surface of the earth at the North Pole, our universe began in a rounded-off, smeared-out, quantum state that precluded an initial singularity.

So what lies at the bottom of a black hole? Not an infinitely compressed singularity, we would expect Hawking and other Quantum Cosmologists to say, merely a great mass compressed to finite Planck dimensions. But Hawking and others are not consistent about this.

The preceding arguments from quantum effects against singularities are exceptionally powerful, but cosmologists like Hawking who rely upon them really do not consistently believe them! Hawking frequently changes his mind on many issues and is very difficult to pin down. First, considering only relativity theory apart from quantum theory, he and Roger Penrose proved that closed universes must begin with initial singularities. Then in A Brief History of Time, Hawking repudiated initial singularities by appealing to quantum effects, presumably indefiniteness and discreteness, and to quantum gravity. In his very recent debate with Penrose in The Nature of Space and Time, 1996, Hawking switched again; expressly repudiating quantum discreteness, Hawking says that he sees "no reason to abandon the continuum theories that have been so successful,",38 and he affirms again that the Big Bang emerged from a singularity. ${ }^{39}$

Hawking and Penrose both believe that black holes, collapsing neutron stars, and collapsing universes regularly coalesce into real singularities below Planck dimensions. They agree that "cosmic censorship" hides the singularities in black holes and neutron stars from our view because they are cloaked to outside observers by their surrounding event horizon, ${ }^{40}$ but the singularity of the Big Bang is "naked" and exposed to us. In The Nature of Space and Time, Hawking says that "Cosmic censorship may shield us from black hole singularities but we see the big bang in full frontal nakedness."

One of Hawking's hands does not seem to know what the other hand is doing! Hawking appears to accept arguments from quantum effects against singularities when convenient, and to reject them when convenient! But we must allow him to change his mind. Penrose, by contrast, has never ruled out an initial singularity or appealed to quantum effects like discreteness and indefiniteness to exclude it. Penrose persuaded Hawking that the distinctness of an initial Big Bang involves a different kind of quantum effect, a peculiar sort of spatial curvature, based ultimately on quantum gravity. ${ }^{42}$

As Penrose explains, there is a fundamental difference between an initial world-creating singularity and the terminal singularities that appear in black holes or a collapsed universe, but it does not involve such quantum effects as indefiniteness and discreteness. The difference is in the kind of spatial curvature that each requires and manifests. Astrophysics distinguishes two fundamental kinds of spatial curvature, first, that involved in the increasing volume of a small 
sphere being influenced by gravity (called "Ricci curvature"), next, that involved in tidal distortions of this sphere (called "Weyl curvature"). ${ }^{43}$ In the initial world-making singularity, "The Weyl tensor was exactly zero at the big bang itself, while the Ricci curvature diverged to infinity." ${ }^{\prime 44}$ By contrast, in the singularity of a terminal black hole or collapsed universe, the reverse is true: Weyl curvature is infinite and dominates over Ricci curvature. This explains why entropy or disorder is low in an initial Big Bang singularity and high in a Big Crunch singularity, and why time and the Second Law of Thermodynamics would not be reversed in a closed collapsing universe. ${ }^{45}$ An initial singularity is infinitely ordered, completely free from quantum distortions or irregularities in space-time geometry with respect to Weyl curvature; and a terminal singularity is infinitely disordered in this respect, if Penrose is right.

Both Penrose and Hawking now believe that ripples that would tear everything apart would pervade the contracting phase of a collapsing universe. This is how its entropy would continue to increase. Quantum effects like indefiniteness and discreteness begin only when an initial singularity expands to Planck dimensions. ${ }^{46}$ Despite quantum effects, our universe, the only one we know to exist, really could have begun in an initial singularity of nothingness. If Penrose is right, an initial singularity is different, improbable, but not impossible; but it could not be the product of an antecedent collapsing universe with ever-increasing entropy. Singularities still have serious shortcomings.

Quantum effects like discreteness and indefiniteness together with Weyl curvature should rule out singularities in collapsed universes if not also in black holes and dimensionless particles. Hawking, who affirms them, assumes at times that they do not rule out singularities as such. The Hawking who thinks that quantum effects would rule out an initial singularity still affirms singularities in gravitationally collapsed black holes and collapsed neutron stars. In them, presumably, mass/energy could retain sufficient definiteness, continuity, and freedom from distortion to collapse to zero size, despite any and all quantum effects. In them, mass/energy could gradually shrink continuously below Planck dimensions. In them, singularities are physically attainable, so the laws of both classical and quantum physics could apply all the way back to $\mathrm{T}=0$.

Why, then, are initial singularities impossible? Large exceptions should make one suspicious. Singularities may be impossible only when some cosmologists do not want to find them! If black holes and gravitationally collapsing neutron stars can shrink smaller and smaller to singularities, then quantum effects like indefiniteness and discreteness do not rule out singularities as such. According to Penrose, the absence of Weyl curvature from an initial singularity is what makes it infinitely ordered and free from entropy; its presence in a terminal singularity makes it infinitely disordered and riddled with entropy. But this should rule out Oscillationism, for antecedent universes all end with overwhelming entropy. 
Hawking's arbitrariness in rejecting initial singularities, at least for a time, may be seen in his best selling book, A Brief History of Time. In explaining his position, Hawking makes the following contradictory claims about singularities.

1. "There was in fact no singularity at the beginning of the universe."47

2. "Only if we could picture the universe in terms of imaginary time would there be no singularities." ${ }^{.48}$

3. "When one goes back to the real time in which we live, however, there will still appear to be singularities. The poor astronaut who falls into a black hole will still come to a sticky end; only if he lived in imaginary time would he encounter no singularities." 49

Since 1 . is presumably about the real time in which we live, it contradicts 3. Hawking's discussions often fail to clarify the role of quantum mechanics in permitting or excluding singularities, whether initial or terminal. His occasional retreat into an instrumentalist position, according to which physical theories are merely mathematical models that exist only in our minds but tell us nothing about reality, ${ }^{50}$ also contributes nothing to the clarity of his views. His renewed discussion of real versus imaginary time in his 1993 book Black Holes and Baby Universes and Other Essays did little to elucidate his position. ${ }^{51}$

A boundary condition is a state out of which another state arises, normally, a cause. Hawking's peculiar brand of Quantum Cosmology affirms that the ultimate boundary condition for the universe is that there is no ultimate boundary condition; but the meaning of this is extremely obscure. ${ }^{52}$ Carl Sagan took it to mean that the universe has no cause, hence there is nothing for a Creator God to do ${ }^{53}$ Neither Sagan nor anyone else noticed, but this also means that there is nothing for an antecedent universe to do! In one place, writing of a universe "without boundaries or singularities, "54 Hawking seems to regard the terms as synonymns. So regarded, a universe without boundaries is just a universe that does not begin with a singularity, which is perfectly compatible with that variety of quantum oscillationism that dispenses with singularities but not with antecedent universes. Thus, our universe could be bounded by a collapsing temporally antecedent universe from which it bounced, one that crunched only to finite quantum dimensions but not to infinite singularity dimensions, while not being bounded by a singularity. It could be singularity unbounded but temporally bounded-in an extended sense of time that transcends our cosmic epoch.

Robert Jastrow interprets Hawking's no boundary condition to be just another version of Oscillation Cosmology. Any quantum Big Bounce Oscillation Cosmology with an infinite number of cosmic epochs not separated by singularities lacks ultimate boundary conditions (singularities) while having ultimate boundary conditions (causally efficacious antecedent universes). According to Jastrow, Hawking's "universe without a boundary means a 'closed' universe-one which oscillates between expansion and contraction, instead of expanding forever." "ss Jastrow's interpretation of Hawking is not 
entirely baseless. In comparing the Big Bang to a black hole in his Black Holes and Baby Universes and Other Essays, Hawking says that "It may be that there was an earlier phase of the universe in which matter collapsed, to be re-created in the big bang;",56 and many things that Hawking says in 1996 in his and Penrose's The Nature of Space and Time about the Big Crunch and a closed universe sound very much like Oscillationism. ${ }^{57}$ An important analogy between black holes and the Big Bang is that black holes might sometimes explode! They don't always just radiate and slowly fade away. ${ }^{58}$ However, as caution warns, in no instance has anyone ever observed the explosion of a singularity; and if no laws of physics apply to them, we cannot appeal to the physical laws we know to explain how singularities ever could explode.

In developing his own view that the universe has no ultimate boundary, Stephen Hawking compares spacetime to the spherical surface of the earth, but the analogy is not very illuminating. In this comparison, spacetime begins at the North Pole, expands to its maximum at the equator, and then shrinks toward the South Pole. ${ }^{59}$ There, presumably, the process reverses and is repeated over and over again, though this is not perfectly clear. Hawking says that he agrees with St. Augustine that time begins with the creation of the universe, and that it makes no sense to ask what happened before that. ${ }^{60}$

Yet, if the North to South-South to North-phases repeat themselves, the whole of time cannot be contained entirely within a single North to South sweep, unless North represents the absolute boundary condition of the creation of the universe ex nihilo, as it did for St. Augustine. This would rule out not just singularities but also temporally antecedent universes. Without an absolute temporal boundary, a South to North sweep could come before a North to South sweep; and the indefinitely large South Pole of one sweep could be identical with the North Pole of its successor; if so, time as such cannot begin absolutely at any given North Pole, and Hawking is an Oscillationist, as Jastrow claims. No ultimate temporal boundary condition is real if time already existed before our time, our sweep, began. Thus, if there was no time before our time began-which Hawking sometimes affirms, there was a time before our time began-which he also sometimes affirms; and an ultimate boundary condition exists after all! Hawking's position is puzzling if not riddled with flip-flops and contradictions. Like Hawking, many cosmologists cannot make up their minds, equivocate on the meaning of basic concepts ("singularity" versus "temporality" as the meaning of "boundary condition"), often change their minds, and occasionally argue for incompatible positions. Given the complexity of their subject matter, this is easily understandable.

Hawking may or may not be a real Oscillationist. Perhaps his universe without boundary conditions does not oscillate because time is unreal. At times, Hawking seriously doubts the reality of time, something that a true Oscillationist cannot do. Perhaps he confuses the absence of a singularity boundary with the absence of a temporal boundary; but perhaps he wishes to deny the reality of 
time altogether. Imaginary time, favored by Hawking, is really a spatialization of time which, by a mathematical trick, converts time to a fourth spatial dimension. Just what this means is extremely obscure. Hawking explains that imaginary time is "at right angles" to real time; but it is difficult to see how something that is not spatial could be "at right angles" to anything, or how there can be a singularity at the beginning of real time but no singularity in the imaginary time that is also somehow real and at right angles to it. Also, a fourth spatial dimension gets lost in Hawking's analogy with an expanding and contracting globe because these expansions and their cosmic evolutions are temporal.

In purely spatial terms, the North Pole, no matter how inexact, definitely represents a boundary between the earth and its atmosphere; so Hawking's analogy does not adequately illustrate the notion of finite but unbounded time; perhaps no analogy could succeed. If time really can be spatialized, Hawking's initial temporal singularity is avoided by eliminating time altogether. Once time is abolished, the whole universe is timeless; and our experience of time is an illusion. But why isn't space also an illusion? A purely spatial but timeless Hawking universe is no longer our universe, no longer a quantum universe in which time and space are inseparable but not identical. Hawking pays a very high price for avoiding an initial singularity. His theory becomes irrelevant to our world. As Benjamin Franklin said, "Time is the stuff life is made of." The real world of nature is temporal through and through. Contemporary physics does not spatialize time; it temporalizes space, despite many claims to the contrary. ${ }^{61}$

\section{Critique of Oscillation Cosmology}

Oscillation Cosmologies have troublesome defects. Their most serious flaws must be identified and considered. The greatest difficulties for all Antecedent Universe Cosmologies, including Oscillation Cosmology, are that we do not and cannot directly experience antecedent worlds; we have no inductive empirical access to them because inductive logic is inapplicable where we know only one of a kind; and the laws used to reason back to antecedent worlds break down before they take us that far. Science cannot establish their existence or know their essence. Other difficulties for Oscillation Cosmologies center upon A. their affirmation that space is finite with sufficient mass/energy to open and close it an infinite number of times, B. their treatment of singularities, $C$. their affirmation of universal causation, D. their appeal to quantum effects, and $\mathrm{E}$. their approach to thermodynamics.

\section{A. An Infinite Number of Closed Universes}

Oscillation Cosmologists do not agree with Plasma Cosmology that space is infinite, but they share its view that time is infinite. Only a finite quantity of 
spatially extended stuff expands and contracts forever in oscillating universes. If singularities are rejected, oscillating universes have a finite mass/energy density and both a minimum and a maximum magnitude that are finite. When maximum allowable magnitude is reached, expansion ceases; and a Big Squeeze commences. When the allowed minimum is reached, contraction ceases, and another Big Bang begins. And this goes on forever.

Unlike Gamow's One-Bang theory, Oscillation Cosmologies assume that enough mass/energy existed in an infinite number of spatially finite consecutive universes to close them all and prevent them from expanding forever, no matter how much they differ otherwise with respect to laws, physical constants, and initial conditions. The requirement that all antecedent universes be closed places severe limits on admissible laws, constants, and initial conditions for all oscillating universes. It excludes an immense number of possible laws, constants, and initial conditions-all those that would engender flatness or openness. If only one universe in an oscillating series is flat or open, the series breaks and absolutely terminates. Oscillationists cannot explain why an immense number of logically possible open or flat universes are metaphysically impossible, or why an infinite number of only closed universes can or must exist.

Open and flat universes never collapse and can have no Big Crunch successors. If a prior universe in our own presumed series had been open or flat, our world would have been impossible. For Oscillationism, necessary conditions for the origin and existence of our universe are fulfilled only if all earlier cosmic epochs were closed.

Consider this very forceful argument against an infinite series of oscillating universes. Many cosmologists are convinced that a Big Crunch will so diversify initial conditions for its successor that eventually, given enough time, an open universe or epoch having no successors will inevitably occur. John Barrow and M. P. Dabrowski think that they understand the mechanism by which some if not all series of oscillating universes will eventually end in openness: the maximum size of each epoch increases with increasing entropy; and eventually they become so large that oscillations cease and expansion continues forever. ${ }^{62}$ If this is possible at all, then no oscillating set of universes could be eternal, especially if, as some hold, all possibilities are actual.

By definition, no open or flat universe ends with a Big Crunch; thus, no open or flat universe can have a successor universe that rebounds from its Big Crunch. Mechanisms may exist by which any and every infinite set of universes will eventually produce and thus terminate with an open or flat universe. This would definitely happen if every temporally ordered infinite set of worlds diversifies to actualize every possible universe, as Atheistic and Finalistic Anthropic Cosmologists propose.

If an open or flat universe will come along eventually by accident in an infinite set of cosmic epochs actualizing all possible worlds, then every world in such a set must be open, for every member has an infinite number of prede- 
cessors. The predecessors of every universe in an infinitely prolonged set collectively endure for an infinite amount of time, which is quite long enough to engender openness, given the presumption of infinite diversification within infinite time. Thus every infinite oscillating series of universes must end with an open or flat universe; indeed, every individual universe will be open or flat because in an infinite series each would have an infinite number of predecessors; so the very idea of oscillationism is nonsense!

Closed universes must be finite in space and mass/energy. Robert $\mathbf{J}$. Russell suggests that an open universe is actually infinite in size and mass/ energy, ${ }^{63}$ but in an important sense this is not true. If spatially infinite when maximally expanded, an oscillating universe that contracts at a finite temporal rate would never totally collapse because finitude cannot use up infinity. Similarly, and for the same basic reason, a universe that is initially finite and expands at a finite rate could never achieve spatial infinity.

Both closed and open universes must be actually finite, but open universes have an indefinitely large potential for expansion. In this sense they are finite but unbounded; their actuality is finite, but they are unbounded with respect to their potentiality for expansion. If an open universe begins with finite space/ energy and expands at a finite rate, it will still be finite after fifteen billion years-and after fifteen billion billion years; only its potential for future expansion is infinite, never its actuality.

Oscillation Cosmologists must insist that in an infinite number of actual universes, including our own, Omega (total density) is greater than 1 (perfect balance, or critical density). Obviously, this cannot be verified; and our universe with an increasing rate of Hubble expansion, and with total density of from .1 to .3 or .4 at most, appears to be open. Even a flat universe with an Omega density of exactly 1 is incompatible with Oscillationism because, like open universes, flat universes never collapse and never have successors.

Recall that the case against the existence of an enormous quantity of dark or hidden cosmic mass that cannot be located is very strong. Astronomers can find only ten to thirty percent or so of the mass/energy required to close the universe, despite their best efforts. After devoting much of his book on The Dark Side of the Universe to examining the case for and against dark matter, James Trefel concluded:

It used to be customary in discussions of this sort to entertain the idea that the universe was cyclical-that the Big Bang would be followed by a collapse (the Big Crunch) and another expansion (the Big Bounce). But if our current ideas are true, this will not happen. The Universe has one shot at existence-one explosion followed by an expansion that slows down for an infinite length of time. ${ }^{64}$ 
Yes, some dramatic discovery tomorrow might reveal the existence of the missing mass; scientists are constantly stumbling upon the unexpected, especially with the aid of the Hubble Space Telescope. New sources of both luminous and cold dark matter are regularly identified; but will we ever locate seventy to ninety percent more? Will astronomers or particle physicist ever find enough ordinary matter or previously undetected cold dark matter to close the universe? Maybe so. Maybe not. The existence of enough mass to close the universe is merely a "Philosophical Maybe," an abstruse possibility lacking probability, given our vantage point in history. To every "Philosophical Maybe," corresponds a "Maybe Not." In the realm of pure possibilities, "possibly so" is always checkmated by "possibly not." We should not be intimidated by mere possibilities, and we should never confuse possibilities with probabilities.

Given the present state of human knowledge, it is reasonable to think that enough mass/energy probably does not exist to close the universe even once, much less an infinite number of previous times. From seventy to ninety percent is missing; the odds against finding enough are just too great; and the expanding rate of cosmic expansion seems to rule it out definitively.

As explained in Chapter Three, the recent discovery that the rate of cosmic expansion is increasing, not slowing, as earlier assumed (for example, by Trefel above), counts overwhelmingly against a closed universe. Too little mass exists in the universe to slow down its expansion rate, much less to stop it. Yet, oscillating universes must be closed and reopened an infinite number of times, no matter how different other epochs are from our own. For exceptionally good reasons, we should reject the oscillation hypothesis. We really do not know that an antecedent Big Crunch happened even once, much less an infinite number of times. What we do know suggests that it never happened at all. We will return later to the problem of the missing mass.

\section{B. Singularities vs. Finite Size Maximal Compression States}

A few Oscillation Cosmologists like a younger John A. Wheeler and a contemporary John Gribbin maintain that each cosmic epoch begins and ends with a singularity, a state of infinite compression, infinite density, infinite heat, infinitesimal size, and infinite curvature. Other Oscillation Cosmologists like Mark Israelit and Nathan Rosen ${ }^{65}$ repudiate infinitesimally small initial singularities and contend that the maximal compression state of the antecedent universe was finite in size or volume. Let us further considers rebounds with and without singularities.

\section{i. Rebounds from Singularities?}

The reality of an initial singularity from which an oscillating universe could rebound may be doubted for a variety of reasons. For one thing, there are 
serious problems about its empirical status. I. L. Rozental says that "The singularity situation in cosmology is in contradiction to all accumulated physical experience." ${ }^{166}$ According to Rozental, singularities cannot be created in the laboratory because "under terrestrial conditions" a phase transition always occurs as pressure approaches infinity, and "the singular state is not achieved."67 The idea that all sensory experience counts against singularities can be further expanded. It is absolutely impossible for our physical senses to perceive directly anything that has no size or spatial magnitude whatsoever. Conceptually constructed Euclidean points and spatiotemporal singularities are so small that they are indeed empirically nothing, and the claim that our universe was created out of an initial singularity is empirically indistinguishable from the claim that it was created ex nihilo.

An initial singularity for our universe could not have been perceived directly if we had been there, but confidence in an initial singularity may be inductively grounded in some other way. Ignoring quantum effects for the moment, perhaps an initial singularity can be extrapolated mathematically from observable processes such as increasing entropy, the redshift, and the Hubble expansion. If these processes are reversed and traced far enough into the past, in ten to twenty billion years, the results equal 0 . This assumes that the laws and basic structures of nature do not break down before zero diameter or magnitude is reached, that abstract calculations can accurately reflect physical processes that contract all the way down to zero and not just up to the edge of it, and that quantum physics does not block the descent to nothingness.

If, as most quantum physicists believe, the laws of nature do not break down at Planck spacetime but nevertheless become inapplicable below Planck dimensions, it would definitely not be rational to believe that our universe began in a singularity at $T=0$. Quantum Cosmology indicates that the laws and the most basic concepts that we apply to nature do become inapplicable to spacetime below Planck's Wall because nothing physical whatsoever can exist on its other side. Scientifically, we can trace origins back no further than when the universe was $10^{-43}\left(1 / 10^{43}\right)$ of a second old and its size was $10^{-33}\left(1 / 10^{33}\right)$ of a centimeter in diameter. Quantum Cosmologists like I. L. Rozental and Stephen Hawking (at times) are convinced that quantum effects make collapses to initial singularities impossible (despite the fact that Hawking still believes in black holes with singularities at their core). We will soon see that Quantum Cosmologies have serious problems of their own.

Because they suggest creation ex nihilo, singularities create an interesting philosophical problem for Oscillationism and all other naturalistic Antecedent Universe Cosmologies. Theistic creation ex nihilo affirms the known existence of only one universe and is not vulnerable to this difficulty. Its proponents would be quite happy if modern science discovers that the universe was created out of nothing in the finite past! 
The philosophical difficulty is this. Naturalistic metaphysics maintains that our universe has existed throughout an infinite past; but the notion of "our universe" is not easily stretched to infinity, despite Oscillation Cosmology. The problem of personal identity through time has as its counterpart the problem of cosmic identity through time. Our cosmic epoch, which began around fifteen billion years ago, could not belong to the same universe as its predecessors if it is separated from them by even one singularity, much less by an infinite number of them. Conceptually, singularities involve the total eradication of space, time, physical causation, continuity, and all laws of nature; so no universe derived from a singularity, including our own, could have any temporal predecessors or physical causal progenitors.

If antecedent Cosmic Epoch A and subsequent Cosmic Epoch B are spatiotemporally and causally linked through a singularity, that is, by causal and spatiotemporal nothingness, then they are not linked at all. Two regions belong to the same universe only if they belong to the same spacetime system, that is, if it is at least theoretically possible to get from one to the other by traveling through space or time; all members of the spacetime system have physical causal relations with-are either the physical cause or effect of-some other members of that system; and all members function statistically in accord with an all-pervasive set of physical laws. "Sameness of universe" thus involves continuity of (1) space, (2) time, (3) physical causation, and (4) natural laws. Our universe is the totality of the spacetime system to which we belong, including all the laws, mass/energy, and cones of physical causation within it. To belong to the same spacetime system, any two things must be bound together lawfully by spatiotemporal continuities, contiguities, and causal processes. Entities belong to our system of spacetime only if spatiotemporal continuities and physical causal bonds link them lawfully to us directly or indirectly.

No lawful spatiotemporal and causal continuities whatsoever obtain between universes separated by singularities, where all of the above break down and disappear. Without something physical (spatiotemporal) there is no physical causation. Since physical causation and the laws of nature break down in a singularity, no universe separated from another by a singularity could be its physical cause or project its mass/energy and its laws into its successor. An antecedent universe that crunched to a singularity could not be the cause of our Big Bang.

In fact, it could not even be antecedent! Our world could not be part of an eternal universe, as Naturalists hold, if it began fifteen billion years ago and is separated from its antecedents by singularities. Singularities eliminate temporal as well as causal continuity and succession, so speaking of antecedent universes separated by singularities makes no sense. No temporal relations can exist between two cosmic epochs separated by utter nontemporality.

Nothing can be earlier than the first moment of time. If our universe begins and ends with a singularity, nothing could precede it or follow it. The Big Bang 
would be the beginning of time itself; nothing could antedate it. One universe can precede another only if they belong to the same spatiotemporal series. If singularities separate them, the spatiotemporal series is unconditionally interrupted; and the notion of "antecedent universes" becomes inapplicable and unintelligible. Antecedent Universe Cosmologies that affirm singularities are logically incoherent, and Oscillation Cosmologists must avoid them.

A naturalistic metaphysics that accepts singularities between cosmic epochs is itself incoherent. Our universe cannot be separated from its predecessor by a singularity because applying the concept of "predecessor" to such a relationship is unintelligible. Big Bang Cosmology shows that our universe is not eternal, contrary to the assumptions of Naturalism. If some universes are eternal, they can have no spatiotemporal or causal continuity with our own through singularities; and we can never know of their existence on lawful, inductive, or scientific grounds.

On non-scientific grounds, many theologians postulate Heaven and Hell as completely independent spacetime systems, that is, as Other Worlds, that have no direct spatiotemporal or causal relations with our own. Naturalists who accept singularities incoherently embrace disconnected, transcendent, OtherWorldly spacetime systems-the very things that they repudiate so vehement when quarreling with theologians about Heaven and Hell! Our world is not eternal and could not be connected to an everlasting series of worlds if it began fifteen billion years ago in a singularity of nothingness.

If and when a singularity forms at the end of a "Big Crunch," why doesn't it just stay there forever? We actually have neither empirical nor theoretical evidence that they ever explode, and this is absolutely devastating to any Oscillationism that separates universes by singularities or derives them from singularities. In no instance has a singularity ever been observed to explode. Even theoretically, no one knows what would cause a singularity to explode since no known laws of physics apply to them. According to Alan H. Guth, Oscillationism is now very unpopular with most scientific-minded cosmologists for this very reason. Guth indicates that what we know of gravity in relativity theory "does not allow a crunching universe to bounce into a big bang," but, he insists,

This is not a fatal objection, however, since general relativity presumably breaks down at the extraordinarily high densities encountered in a big crunch. Nonetheless, since there is no reliable theory that describes how a universe might bounce, the basis of the oscillating universe theory relies solely on speculation. ${ }^{68}$

Since all natural laws break down in singularities, no known or knowable laws of nature could permit them to explode. Something very similar is true with respect to big bounces from finite compaction states. Given our ignorance with 
respect to high density physics, no known laws of physics could permit them to explode. As Martin Rees indicates, "Physical conditions in the 'bounce' would transcend the physics we understand, so that nothing could be said about the possibility of a rebound into a new cycle-still less about what memory would be preserved of what had gone before." ${ }^{\prime 9}$ With or without singularities, quantum fluctuations really could not do the job, for we really know almost nothing about quantum fluctuations in high density physics. Inflation Cosmology does not derive its many worlds from exploding singularities or immensely compressed antecedent universes. Inflation, which does not appeal to explosions at all, requires just the right kind of diluted quantum-foamy "empty space"; and crunched-up antecedent universes just aren't the right stuff?

\section{ii. Big Bounces Without Singularities}

Singularities are not easily circumvented, but Oscillation Cosmology can be developed without them. If antecedent cosmic epochs are not infinitely compacted into singularities as they crunch to an end, if they rebound from finite size maximal compression states, some of the foregoing difficulties can be avoided, but at a price. If the laws of nature as we know them apply to high density situations, corridors of no more than Planck dimensions might link universes causally, spatially, and temporally and provide for the continuation of natural laws from epoch to epoch.

Some Antecedent Universe Cosmologists explicitly reject singularities and conjecture that antecedent universes rebound while still finite in diameter, curvature, density, and temperature, thus avoiding the embarrassing infinities and breakdowns of singularities. These minimal-size-maximal-compression states may be quite large. Georges Lemaitre initiated Oscillation Cosmology with his image of the Phoenix that dies a fiery death, then rises again from its own ashes. His "primeval atom" was two hundred million miles in diameter when it exploded. ${ }^{70}$ George Gamow speculated that when the previous universe crunched to its maximal pre-expansion density, all of the matter within the reach of a 200-inch telescope "must have occupied a sphere only thirty times as large as the sun."' Eric Lerner's Mini-Bang occurred when an antecedent metagalaxy collapsed to a hundred million light years across. ${ }^{72} \mathrm{~A}$. Karel Velan thinks that before a Big Bang explosion, a collapsed universe has a radius of $1.17 \times 10^{14}$ $\mathrm{cm},{ }^{73}$ that is, 1.17 trillion centimeters. Most Antecedent Universe Cosmologists actually prefer sub-microscopic but still finite dimensions as minimal-size, maximal-compression states for collapsing/erupting universes, partly because they think that at lesser volumes and densities, thermonuclear reactions would not be sufficiently hot and compact to destroy heavy elements and replenish the universe's supplies of hydrogen and helium. Defenders of large finite maximalcompression rebounds may find it difficult to explain the physics of hydrogen renewal. 
Tiny Planck spacetime is the most popular minimal-size-maximal-compression dimension for contracting/rebounding universes, or for any physical realities at all. ${ }^{74}$ Contracting universes may proceed almost to zero, but not quite, before a Big Bounce erupts to recreate another world.

Oscillationists must answer this question: What initiates the explosion? What makes a contracting universe bounce? Quantum fluctuations might make Big Bounces inevitable, but only if the laws of quantum physics obtain in all antecedent universes and at the junctures between universes, and only if all logically possible non-quantum universes are inexplicably non-existent. With Big Bounces, Planck size threads could connect cosmic epochs; but these are so minute that one still wonders if anything is left of the notion of same universe. All ordinary sameness is left far behind long before we arrive at Planck's Wall. From a commonsense standpoint, a Big Bang universe would be a distinct universe whether or not it issues from a singularity or a Planck size quantum corridor. Yet, some Oscillationists submit, the connecting link might not be implausibly thin.

\section{Singularities and Universal Physical Causation}

Perhaps singularities are ruled out because they are incompatible with Naturalism's metaphysical causal principle: "All events have natural causes." In 1965, the year that the omnipresent background radiation was discovered, Milton Munitz rejected an initial singularity for our universe for this reason; but his arguments confused scientific methodology with Naturalism's principle of physical causation.

Accounts of origins must transcend finite limits like those set by an initial singularity, according to Munitz, because scientific method presupposes that all spatiotemporal events are caused by other spatiotemporal events. An original event proceeding from the nothingness of a singularity would violate this methodological rule, (which is really a metaphysical rule in disguise), so science itself is incompatible with an initial singularity. According to Munitz, it is always possible to find "some more refined theory, in which inferences would be made to events even earlier than the one identified as "the beginning" in the theory of coarser grain." ${ }^{\prime \prime}$ As he expressed this argument in The Mystery of Existence,

Science is grounded in the use of the Principle of Sufficient Reason and, therefore, always leaves open the possibility of finding the explanation of any event. To say there is some unique event, marking the beginning of the universe for which no explanation can be given, is to say something contrary to the method of science. It is for this reason, I should argue, that any conception of the beginning of the universe, when defended under the aegis of some supposedly scientific cosmology, is an indefensible notion. ${ }^{76}$ 
Here Munitz clearly identifies the Principle of Sufficient Reason with the naturalistic metaphysical principle that "All natural events have natural causes;" otherwise, the door would be open for the Theist who thinks that God's original act of creation is the sufficient reason for the existence of the world. A natural cause belongs to the same system of spacetime as its natural effect; but God presumably transcends the spacetime of our world; and so do all antecedent universes. Munitz has natural causes in mind when he says that "All events investigated by science are ones for which it is relevant to inquire into their causal conditions" and that "On scientific grounds, one could never hope to establish that the universe had an absolute origin, or came into existence." Here Munitz assumes that a scientific inquiry into natural causes will always find them-ad infinitum; for this reason, he assumes, science could never discover absolute origination.

Munitz is mistaken in thinking that science could never discover an absolute origin for the universe merely because it must always inquire about the natural causal conditions for everything that it investigates. Methodology is confused with metaphysics. Scientific methodology always inquires about natural causes, but only a non-empirical Naturalistic metaphysics guarantees that inquirers will always find that for which they are looking. Scientific methodology needs only the imperative: "Look for natural causes!" It does not require an a priori metaphysical guarantee of success.

When divorced from naturalistic metaphysics, science can conclude that scientific method reaches its iimits with an initial cosmic singularity, if this is indeed where time, space, natural laws, and physical causation commense, the point beyond which they can be traced and applied no further, the end of scientific explanation.

As Robert Jastrow indicated, "Science has its own religion. That religion is founded on faith in natural law and in cause and effect. Science has succeeded in posing, on its own terms, questions not answerable within the domain

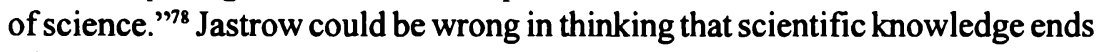
when it stumbles upon an initial singularity; but he correctly judges that any confusion of methodology with metaphysics, like Munitz's, is religiously grounded in a blind faith in absolute natural causation, a faith that empirical scientific methodology alone cannot substantiate. Even quantum physics calls universal causal absolutism into question, as we will see. That an initial singularity would be incompatible with scientific method and universal physical causation is only a minor obstacle for Oscillation Cosmology, but other difficulties are more serious.

\section{Quantum Effects Near Singularities}

We should be very cautious about accepting Quantum Cosmology's supposition that when the whole universe is squeezed down to the size of sub-atomic quan- 
tum objects, it will begin to obey the laws of quantum physics and manifest quantum effects like indefiniteness, discreteness, and spontaneity-if for no other reason than that Quantum Cosmologists seem to believe this only when it is convenient. Stephen Hawking suggests that size is the relevant consideration for appealing to quantum effects when he writes that "There must have been a time in the very early universe when the universe was so small, that one could no longer ignore the small-scale effects of quantum mechanics." ers also treat size as the only relevant consideration in applying quantum physics to the universe as a whole. ${ }^{80}$

The real difficulty is that quantum demeanor may depend on more than or something other than just size. It may depend heavily on degree and kind of curvature, compression, density, temperature, or quantity of energy involved. An entire universe compressed to the size of an electron or quark may be too curved, compressed, dense, or hot to obey quantum laws; or the quantity of energy may be too great. Roger Penrose, who doubts that size and distance mark the boundary between quantum level and classical level events, suggests that all quantum level events involve "very tiny differences in energy." "II If so, an entire universe of energy concentrated to the size of a photon just might be too much for quantum effects!

Without agreeing with them completely, Heinz R. Pagels notes that according to cautious critics, "Theorists exploring, on paper, the very early universe have gone too far. Extrapolating from theories that work in the relatively low-energy domain examined by terrestrial accelerators to such ultrahigh energies is a dubious enterprise. ${ }^{\prime 2}$ Martin Rees recognizes that "...Physics at ultrahigh energies... is almost completely unknown." ${ }^{\text {"3 } 3}$ John Gribbin and Rees warn that "Because the physics of the ultracompressed, high-density stages is speculative, we have no firm understanding of exactly where the fluctuations come from. ${ }^{\text {"84 }}$ This is precisely the difficulty. The physics of ultracompressed highdensity states is largely untested and unknown. As Mark Israelit and Nathan Rosen affirm, "We lack any knowledge whatsoever of the constitution of matter under such extreme conditions", 85 yet they make many assumptions about it. Jonathan J. Halliwell recognizes that "Quantum mechanics was developed to describe atomic-scale phenomena," and he recognizes the enormous contentiousness of "the most extravagant extrapolation possible: that quantum mechanics applies to the entire universe at all times and to everything in it." ${ }^{186}$

None of the sub-atomic particles that we know to be affected by quantum indefiniteness, discreteness, and spontaneity are experienced and tested at anything close to what the curvature, compression, density, temperature, and energy quantity of the universe as a whole would be if compacted to the volume of an atom, electron, photon, quark, or something even smaller. The differences in proportions are truly astronomical, and these enormous differences could easily distort or even completely negate quantum effects. In the absence of experimental confirmation, there is room for serious doubt that the whole 
universe obeys the laws of quantum physics when compressed to Planck (or smaller) dimensions. Every law of nature that takes us back to "the beginning" may be inapplicable sufficiently close to $\mathrm{T}=0$, which is why Scientific Cosmological Agnostics proclaim that we just can't know scientifically what caused the Big Bang. Neither caution nor radical skepticism about the applicability of the laws of quantum physics to conditions at or near the origin of the universe will be popular with many contemporary scientific cosmologists, but perhaps they should reconsider.

Without quantum effects at the beginning of creation, Oscillation Cosmologies are indefensible because they cannot otherwise avoid singularities. With quantum effects, Oscillation Cosmologies are still on shaky ground for reasons already given and for others to follow. We must now examine some of the logical and philosophical implications of these options, even though we have serious doubts about the applicability of quantum laws to an ultracompressed universe.

\section{E. Oscillationism and Thermodynamics}

Oscillation Cosmologies presuppose the existence of sufficient mass/energy in all cosmic epochs to reverse their expansion and close them down, no matter how different their contents, laws, constants, and initial conditions might be. Our own cosmic epoch seems to be very much out of place within such an endless series of oscillating epochs. Our universe lacks seventy percent or more of the mass required for reversal. Oscillationists may have a strong faith that the missing mass is really there; but they cannot prove it, and it has now been decisively disproved by the discovery in 1998 that the pace of cosmic expansion is accelerating rather than slowing. This could not be true of a universe that will eventually cease expanding and gradually collapse into a Big Crunch. Oscillation Cosmologists cannot reconcile their robust Omega $=1+$ requirement with the paltry Omega $=.1$ to .3 of our actual universe as we know it.

They might resort to the desperate strategy of contending that either the First Law of Thermodynamics or the Law of Gravity were grossly violated at the beginning of our eccentric cosmic epoch, even though they held in all antecedent universes. They might argue that enough mass or gravity was present in an infinite number of antecedent universes to close them, but our exceptional universe is open because huge quantities of mass/energy or gravitational attraction just disappeared somehow at the beginning of our atypical cosmic epoch. Perhaps so. Perhaps not. If this happened, Oscillationists must explain how and why; but no explanation is readily forthcoming. In an infinite set of successive universes, why is ours an exception to the rules?

If a singularity preceded our epoch, the notion of antecedent universes is meaningless; but without an initial singularity to reshuffle everything drastically, we have no good reason to believe that any physical fundamentals change 
dramatically from epoch to epoch. Natural laws, including the First and Second Laws of Thermodynamics, would not break down between epochs that are not separated by singularities. Oscillationists have no adequate theory or mechanism to explain an enormous one-time extinction of mass or weakening of gravity. With no initial singularity, the First Law of Thermodynamics, the conservation of mass/energy, weighs heavily against significant extinctions of mass and gravitation between epochs. An implausible theory cannot be saved by appeal to an even more implausible theory.

The Second Law of Thermodynamics, the law of increasing entropy or disorder, also makes serious trouble for Oscillation Cosmologies, even without singularities. Cosmologists like Paul Davies ${ }^{87}$ and Alan Lightman suggest that it does ${ }^{88}$ Others like Alan MacRobert, ${ }^{89}$ Steven Weinberg, ${ }^{90}$ Ya. B. Zel'Dovich and I. D. Novikov, ${ }^{91}$ James Peebles, ${ }^{92}$ and Alan Guth ${ }^{93}$ reject Oscillationism outright because they are convinced that the Second Law of increasing entropy or disorder excludes it. Endless oscillations and divisions are ruled out completely by increasing entropy. S. A. Bludman argued in 1984 that

because of the huge entropy generated in our Universe, far from oscillating, a closed universe can go through one cycle of expansion and contraction. Whether closed or open, reversing or monotonically expanding, the severely irreversible phase transitions transpiring give the Universe a definite beginning, middle and end the ultimate crunch can never be reversed. Nor could it have bounced in the past if it began hot or developed a great deal of entropy in a first contraction. ${ }^{94}$

Most scientific cosmologists believe that the law of increasing entropy holds not only during the initial expansion phase but also during the contraction phase of a universe undergoing gravitational collapse. They have no doubt that the law of increasing entropy would apply continuously through successive cosmic epochs, no matter how these are connected. They may or may not be right, but if they are, Oscillation Cosmology is dealt a deadly blow.

Since disorder constantly increases, according to the Second Law, our present universe would be infinitely chaotic if it were preceded by an infinite number of cosmic epochs, as Oscillationism maintains. Because our universe is not infinitely chaotic, an infinite number of antecedent universes did not antedate it, and Oscillationism is dead.

Unless Oscillationists can find a way around the Second Law of Thermodynamics, increasing entropy is a formidable obstacle, not only for them, but also for other Antecedent Universe Cosmologists. But the situation may not be completely hopeless.

John A. Wheeler argued in 1973 that when a collapsing universe shrinks to a singularity, a drastic reprocessing of mass, charge, physical constants, natural laws, and all details of the system occurs; and the ensuing universe starts 
out on a completely fresh dynamic cycle. ${ }^{95}$ Singularities might insure that a universe that ends in maximal entropy would be followed by another universe that begins in minimal entropy, but many unresolved problems are hidden in the obscure notion of "reprocessing."

Quantum Cosmologists wanting to dispense with singularities also speculate that when a cosmic epoch shrinks to quantum size, quantum effects wash out all existing entropy, all information about what went before; and thus each new epoch starts afresh. As Andrei Linde recalls, M. A. Markov developed "a model of an eternal, oscillating universe, which at each cycle of its evolution forgets what occurred before." In Markov's own words, "The state of maximum contraction would play a peculiar role of 'purgatory,' purifying the universe from 'excessive' mass and entropy acquired in a previous expansion and contraction." 97 Robert Dicke and P. J. E. Peebles, commenting on oscillating universes, say that "Experience would suggest the total entropy can only increase, though it certainly is conceivable that in the new physics of the bounce, entropy is eliminated, perhaps lost in black holes left over after the big bang." 98 Notable authorities may be quoted on both sides of the question of whether entropy continues from epoch to epoch, but which view is most plausible?

Many cosmologists are convinced that only an initial singularity, if anything, could guarantee a scramble sufficiently thorough to insure the demise of the Second Law of Thermodynamics from epoch to epoch. Understandably, a cosmic epoch would have to forget its composition and structure if it collapses into a singularity, for at that point there is no space, time, physical causation, physical structures, or natural laws. In singularities, and only in them, the laws of nature are gone, and so are all physical causes, constants, fields, masses, particles, and components. Nothing remains to remember or carry forward the disorder inherited from a preceding epoch. Unfortunately, along with entropy, an initial singularity also obliterates all lawful spatial, temporal, and causal continuity between cosmic epochs In a singularity, both the First Law and Second Laws of Thermodynamics are gone, and nothing remains to insure that enough physical energy or orderliness will persist from epoch to epoch to keep the series going.

Most cosmologists think that complete reprocessing would not occur if a universe collapses only to quantum (or larger) size, and the rest of us may just have to take their word for it. At finite dimensions of compaction, something spatial, temporal, causal, and lawful remains of a maximally compressed antecedent universe; and, despite Markov's doubts, just enough probably remains to carry forward the entropy, the distorting Weyl curvature, of a preceding epoch into a later one. If quantum laws and processes are preserved through the passage from one epoch to another, so are the laws of thermodynamics. If cosmologists can't affirm continuing quantum laws without affirming the laws of thermodynamics, the Second Law of Thermodynamics is a major hurdle for Antecedent Universe Cosmologies that appeal to quantum effects to avoid an 
initial singularity, for they can't avoid an increase of entropy from epoch to epoch.

The strategy of resorting to total reprocessing in singularities is extremely risky for Oscillation Cosmology. The physics of the Big Bounce without singularities also requires that all antecedent worlds be closed quantum universes that cannot collapse into singularities because of quantum effects like indefiniteness and discreteness; but in an infinite number of diversifying tries, total reprocessing will insure that at least one rescrambled antecedent universe would be open, flat, and/or non-quantum. Infinite diversification through reprocessing would bring about at least one open or flat universe that breaks the series, and/or one non-quantum universe that ends in a spaceless, timeless, lawless, and causeless singularity-with all its problems.

In an infinite number of antecedent diversifying shuffles that actualize all possibilities, quantum laws and quantum effects themselves would be scrambled out of existence at some point, and so would sufficient mass to close an infinite number of antecedent cosmic epochs. From such antecedent universes, no Big Bounce could occur. The endless chain would be broken; and we would not be here; but here we are! Infinite reprocessing might unscramble entropy, but it would also terminate infinite reprocessing. The non-existence of our world is a very high price to pay for avoiding the Second Law of Thermodynamics. Furthermore, since every epoch in an infinite series has an infinite number of predecessors, every universe must be nonexistent! The very idea of oscillating universes is unintelligible!

\section{i. Entropy Can't Apply to the Universe as a Whole}

Perhaps the Second Law of Thermodynamics does not apply legitimately to the universe as a whole. The philosopher Stephen Toulmin contends that it does not. ${ }^{99}$ The preceding argument against Oscillation Cosmologies based on increasing entropy from epoch to epoch applies the Second Law to the universe as a whole. If Toulmin is right, this application is illegitimate and all objections to Oscillation Cosmologies based on thermodynamics are spurious.

Toulmin's argument is complex, but let's try to go directly to the heart of it. He is concerned in part with the practical implications of the idea that the universe is running down; but his primary focus is on the shift of the Second Law away from applied mechanics, where it properly belongs, to cosmological speculation about the universe as a whole, where he thinks it is out of place. Toulmin believes that this shift is illegitimate because the question "Is the universe as a whole a thermally isolated system?" is senseless. The reason, Toulmin explains, is that

the question how far a given physical system is isolated from its surroundings has a clear enough meaning when asked about any bounded part of 
the universe-being equivalent to the question, to what extent heat exchanges are possible across the boundary-when asked about the universeas-a-whole, its meaning is completely obscure. ${ }^{100}$

We cannot talk intelligibly about heat exchanges across the boundary of the universe as a whole, Toulmin maintains, because nothing exists outside the boundary of the universe as a whole. The universe has no surroundings. ${ }^{101}$ The Second Law of Thermodynamics tells us that disorder increases in thermally isolated systems; it applies intelligibly only when systems have boundaries and surroundings; it applies appropriately only within but not to the universe as a whole. According to Toulmin, we do not apply the law of gravity to the universe as a whole because we realize that nothing exists outside the universe to be attracted by it. Similarly, the Second Law of Thermodynamics should not be applied to the universe as a whole because it has no boundaries or surroundings. ${ }^{102}$

\section{ii. Entropy Can Apply to the Universe as a Whole}

Fortunately, scientists seldom pay much attention when philosophers tell them what they can and cannot do or think. Toulmin's argument is unconvincing. If nothing exists outside the boundaries of the universe as a whole, this means that it is the ultimate isolated system par excellence; as Victor Stenger says, "Only the universe is a completely closed system." ${ }^{\prime \prime 3}$ If the universe as a whole has no surroundings, then it is clearly impossible for anything to affect the constancy of its supply of energy (First Law) or the internal dissipation of its energy and order as it expands (Second Law).

Toulmin's reasons for thinking that the laws of thermodynamics do not apply to the universe as a whole really show that the universe is a perfect example, perhaps the only really perfect example, of a system that can neither gain nor lose energy (once created). Thus, objections to Oscillation Cosmologies based on thermodynamics must be taken seriously after all. If disorder really increases constantly in closed systems, and the universe as a whole is the ultimate closed system, then Oscillation Cosmologies are untenable because they project the entropic universe as a whole infinitely into the past. Our world is not one of infinite chaos; but it would be if the Second Law of Thermodynamics has been operating throughout an infinite past; so Oscillation Cosmologies must be false.

Antecedent Universe Cosmologies clearly affirm that something exists outside a particular cosmic epoch like the one we inhabit, namely, a temporally infinite but imperceptible (to us) larger or older universe. Thus, even on Toulmin's terms, Oscillation Cosmology should have no difficulty applying laws of thermodynamics to our cosmic epoch, which, by hypothesis, is bounded and surrounded by an infinite series of prior cosmic epochs. The real problem is that 
the surroundings of our epoch transmitted little or no entropy into our Big Bang; so they could not be temporally infinite.

Oscillation Cosmologies are doubly cursed. Without singularities between cosmic epochs, the Second Law of Thermodynamics would not break down, and our world would be infinitely chaotic, which it is not. Our orderly cosmic epoch would be impossible if preceded by an infinite number of increasingly disordered universes that dumped their disorder into our Big Bang. With singularities, if the First and Second Laws are periodically reprocessed out of existence, and so is everything else that would make oscillating universes go on forever. Even worse, antecedent universes could have no spatiotemporal or causal connections with later universes; no continuous and infinitely comprehensive temporal series could exist within which one universe could be earlier or later than another.

So, what caused the Big Bang? Oscillation Cosmologies answer that it was caused by an influx of energy from a preceding universe or cosmic epoch. With or without singularities, this answer is incredible and indefensible.

To summarize, Oscillation Cosmologies affirm that our universe, our cosmic epoch, was preceded by an infinite number of antecedent expanding/collapsing universes; but this claim lies far beyond the limits of scientific knowledge. Science cannot establish that even one cosmic epoch preceded our own, much less an infinite number of them. Antecedent universes are supernatural beings that fall outside of our system of spacetime or nature, as Robert Jastrow correctly indicates. They are beings that, by hypothesis, exist before time and outside space, even if it is only our time and space. We know empirically of no universe other than our own; old fashioned Naturalists are right about that! Scientifically, with respect to supernatural spaces and times, we can only be agnostics or Positivists.

Oscillation Cosmologies have additional flaws. They presuppose that our own cosmic epoch contains enough mass/energy to close it and reverse its expansion process, and that this was true of all antecedent universes, an infinite number of them. However, our own cosmic epoch does not seem to be closed. Its expansion rate is increasing, and seventy to ninety percent of the required mass is missing. Maybe we will find it someday; maybe not. We do not know that we will; and the chances are extremely high that we will not, especially now that we know that the Hubble expansion rate of our universe is increasing.

Oscillation Cosmologies also presuppose that all antecedent universes were bouncing quantum universes, but no known laws of physics would cause or allow crunched-up universes to bounce. Oscillationists appeal to periodic reprocessing to eliminate entropy; but this insures that in an infinite number of antecedent diversifying tries, a prior open or flat universe, a non-quantum universe, or a terminal singularity would have resulted from an endless reshuffling of laws and initial conditions; and we and our world would not exist. Yet, 
here we are! Since this consideration applies to every epoch in an infinite series, no worlds at all would exist!

Oscillation Cosmologies differ over whether antecedent collapsing epochs begin and end in singularities, but they are damned if they do and damned if they don't. With singularities, no lawful spatiotemporal or causal relations could link cosmic epochs because space, time, physical causation, and all the laws of nature break down in singularities. The Naturalistic claim that all spatiotemporal events are caused by other spatiotemporal events would be untrue. The very notion of an antecedent universe becomes incoherent and unintelligible. An antecedent universe is postulated as a physical cause of our universe, but physical causation begins after $\mathrm{T}=0$ and cannot be traced back any further. We cannot infer that the cause of our universe was something physical. Singularities having no magnitude at all are empirically indistinguishable from nothingness.

Without singularities, Planck-size or larger spatiotemporal and causal relations could connect successive epochs; but the laws of nature would not break down between epochs; and without a total meltdown, the law of increasing entropy carries over from epoch to epoch. If our epoch was preceded by an infinite number of epochs, and if chaos increased in each and from one to the next, then our epoch would be infinitely chaotic. It is not; so Oscillation Cosmologies must be wrong. Furthermore, if the laws and initial conditions of each universe are totally reprocessed between epochs, an open or flat universe, a non-quantum universe, a terminal singularity, or the extinction of all energy whatsoever would occur in an infinite number of diversifying antecedent tries that actualize all possibilities; and our universe would not exist; but it does.

Quantum effects rescue Oscillation Cosmologies only at the price of unjustifiable favoritism. Quantum indefiniteness and discreteness would exclude all singularities, both initial and those in collapsing neutron stars and black holes. Initial singularities are excluded only in imaginary time, not in real time, if Hawking is right. Quantum effects support neither Oscillation Cosmologies nor even more eccentric Quantum Cosmologies yet to be examined, for we do not know that other worlds exist(ed) as quantum universes, or that atomic or sub-atomic size universes obey the laws of quantum physics when all the energy within them is drastically compacted in size, pressure, density, temperature, and curvature. With or without an initial singularity, our universe is flat or open, thus radically unlike the infinite number of universes that supposedly preceded it; but what happened to the missing gravity or mass?

Oscillation Cosmologies are initially attractive, but their problems are insurmountable. Antecedent Universe Cosmologies are not serious obstacles to theistic belief. This does not establish the truth of theism, but it clears away much of the rubbish that stands in the way. 